\title{
Reproducible Plasmonic Nanopyramid Array of Various Metals for Highly Sensitive Refractometric and Surface-Enhanced Raman Biosensing
}

\author{
Li Zhang, ${ }^{\dagger}$ Xuemeng Li, ${ }^{*}{ }^{\dagger}$ Yangyang Wang, ${ }^{\dagger}$ Kang Sun, ${ }^{\dagger}$ Xuexian Chen, ${ }^{\ddagger}$ Huanjun Chen, ${ }^{\ddagger}$ \\ and Jianhua Zhou*, ${ }^{*}$
}

${ }^{\dagger}$ Key Laboratory of Sensing Technology and Biomedical Instruments of Guangdong Province, School of Engineering and ${ }^{\ddagger}$ State Key Lab of Optoelectronic Materials and Technologies, Guangdong Province Key Laboratory of Display Material and Technology, School of Electronics and Information Technology, Sun Yat-sen University, Guangzhou 510275, China

Supporting Information

\begin{abstract}
Localized surface plasmon resonance (LSPR) biosensors show great potential for practical/commercial use in clinical diagnosis, home healthcare, environmental analysis, and public healthcare. However, two main issues, that is, low refractometric sensitivity and low reproducibility (large-area uniformity and batch-to-batch consistency), hinder the extensive applications of LSPR biosensors. Therefore, plasmonic nanostructures with high sensitivity and excellent reproducibility are desirable for preparing reliable LSPR sensors. Herein, we have fabricated plasmonic nanopyramid arrays (NPAs) for several batches with reproducible morphology and optical properties by elastic soft lithography and metal thermal evaporation. NPAs of various metals
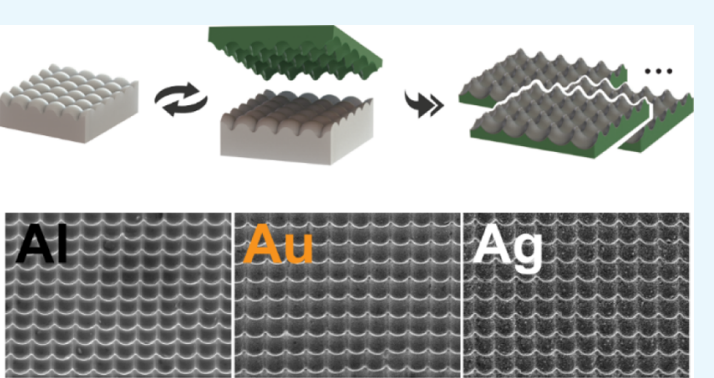
(i.e., $\mathrm{Al}, \mathrm{Au}$, and $\mathrm{Ag}$ ) were also prepared by thermal evaporation with the according metals. The transmission spectra of these NPAs showed several narrow LSPR peaks in the visible-infrared wavelength region. The refractometric sensitivities of the LSPR peaks were systematically studied, and high refractometric sensitivities of 774.0, 472.8, and $421.0 \mathrm{~nm} / \mathrm{RIU}$ were achieved on Al, $\mathrm{Au}$, and Ag NPAs, respectively. To demonstrate the potential of the NPAs for multiplex applications, we first applied this highly sensitive Al NPA biosensor to monitoring the process of proliferation of HeLa cancer cells, in situ and in real time. Then, we demonstrated that the Au NPA was able to identify the absorbed analytes on its surface through the surface-enhanced Raman scattering spectrum. In addition, the finite difference time domain simulations were performed to reveal the electromagnetic field enhancement on NPAs. Because of the properties of high sensitivity and excellent reproducibility of the metal NPA LSPR substrates, as well as the simplicity and cost efficiency of the fabrication method, our proposed work will accelerate the practical use of LSPR sensors.
\end{abstract}

\section{INTRODUCTION}

Plasmonic metal nanostructures have attracted increasing attention because of their wide applications in metamaterials, ${ }^{1,2}$ sunlight harvesting, ${ }^{3,4}$ photocatalyst, ${ }^{5}$ color display, ${ }^{6}$ and optical sensing. ${ }^{7-9}$ Plasmonic biosensors have been appreciated as the advancing and promising optical sensors in recent years because of their properties of ultrasensitivity, high spatial resolution, and label-free detections. ${ }^{10-13}$ Plasmonic biosensors are mainly divided into propagating surface plasmon resonance (PSPR) and localized surface plasmon resonance (LSPR) biosensors. ${ }^{14}$ Compared to PSPR biosensors, LSPR biosensors are remarkable in measuring local refractive index (RI) changes caused by the adsorption of targeted molecules. ${ }^{8,15}$ Moreover, the optical system for LSPR sensing is simpler, whereas SPR system requires coupler (i.e., prism or grating) to activate plasmons. It makes the LSPR sensor more promising in the construction of miniaturized biomedical devices. ${ }^{16,17}$ With the outstanding merits of high spatial resolution, simple optical framework, and capacity of label-free detection, LSPR biosensors show great potential for practical/commercial use in clinical diagnosis, ${ }^{18,19}$ home healthcare, ${ }^{20}$ environmental analysis, $^{21}$ and public healthcare. ${ }^{22}$

However, there are two main issues that hinder the extensive applications of LSPR biosensors, that is, low refractometric sensitivity and low reproducibility (large-area uniformity and batch-to-batch consistency). On the one hand, the refractometric sensitivity of the LSPR biosensor is still low because the LSPRs generally exhibit broad resonance peaks owing to strong radiative damping. ${ }^{23}$ According to the physical principles, LSPR nanostructures exhibiting a shorter decay length and more enhanced electromagnetic field (EF) in a smaller volume than the planar metallic surface can respond better to the local RI (n) changes near the metallic surfaces. To enhance the local EF, many efforts have been made such as changing the size and

Received: December 19, 2017

Accepted: February 8, 2018

Published: October 26, 2018 
shape 24-26 and/or the spatial arrangement $^{27,28}$ of the metal nanoparticles and nanostructures. To obtain largely enhanced EF at confined small sites (so-called "hotspots"), deliberately designed sharp tips, sharp edges, ${ }^{29}$ and narrow gaps between the two nanostructures ${ }^{30}$ on LSPR substrates are highly desirable.

On the other hand, the nanostructures are inhomogeneous in large area and vary from batch to batch. The spot-to-spot and batch-to-batch consistency of the nanostructures is the key to reproducible LSPR response among the measurements of all sensing subsctrates, which is essential for the practical use of LSPR sensors. ${ }^{31}$ There are mainly two conventional approaches to fabricate the LSPR nanostructures: chemical synthesis and physical preparation. In chemical methods, nanocrystals were synthesized in the solution phase and then deposited on a flat substrate to prepare a LSPR sensor. In this case, chemical methods are facing problems of the difficulty in controlling the interdistance between the nanocrystals and eliminating the inconsistency of the nanostructures between different batches. ${ }^{32}$ Compared to chemical synthesis, physical preparation is more promising for LSPR sensors because of the controllable interspace of nanostructures and non-contamination of the metal surface. In physical preparations, e-beam lithography, ${ }^{33}$ focused ion beam lithography, ${ }^{34}$ and photolithography ${ }^{35}$ can realize the precise control of size, shape, and interspace among the metal nanostructures, but they still can not provide low-cost production of large-area nanostructures. Nanosphere lithography, ${ }^{36,37}$ template stripping, ${ }^{38}$ elastic soft lithography (ESL) ${ }^{39}$ are the next-generation approaches that provide cheap production of large-area nanostructures. However, some efforts are still required to obtain a satisfactory LSPR sensor with spot-to-spot and batch-to-batch consistency by these approaches.

Herein, to address these two issues, we proposed a welldesigned metal nanopyramid array (NPA) with high refractometric sensitivity and high reproducibility prepared by ESL and metal thermal evaporation. In the ESL procedures, NPAs with geometries of tapering nanostructure can be easily detached from the template stamp without damage. Therefore, we have fabricated NPAs with excellent reproducibility in large area and in different batches. In the metal thermal evaporation procedure, $\mathrm{Al}, \mathrm{Au}$, and $\mathrm{Ag}$ NPAs were obtained, respectively. Their transmission spectra showed several narrow LSPR peaks in the visible-infrared wavelength region, and the spectra of NPAs from different batches were consistent. The refractometric sensitivities of the LSPR peaks were systematically investigated, and the Al NPA held a highest refractometric sensitivity of $774.0 \mathrm{~nm} / \mathrm{RIU}$. This highly sensitive Al NPA was applied to monitoring the process of proliferation of HeLa cancer cells. In addition, the Au NPA with enhanced local EF was used to provide a surface-enhanced Raman scattering (SERS) spectrum of the 4-methylbenzenethiol (4-MBT) monolayer. Furthermore, the finite difference time domain (FDTD) simulations were performed to investigate the EF enhancement of the NPAs. This work will accelerate the practical process of LSPR sensors on the basis of the simplicity and cost efficiency of the fabrication method, as well as the properties of excellent reproducibility and high sensitivity of the NPA LSPR substrate.

\section{RESULTS AND DISCUSSION}

Fabrication of Reproducible Metal NPAs. Figure 1 shows the scheme of fabricating metal NPAs by two steps: ESL
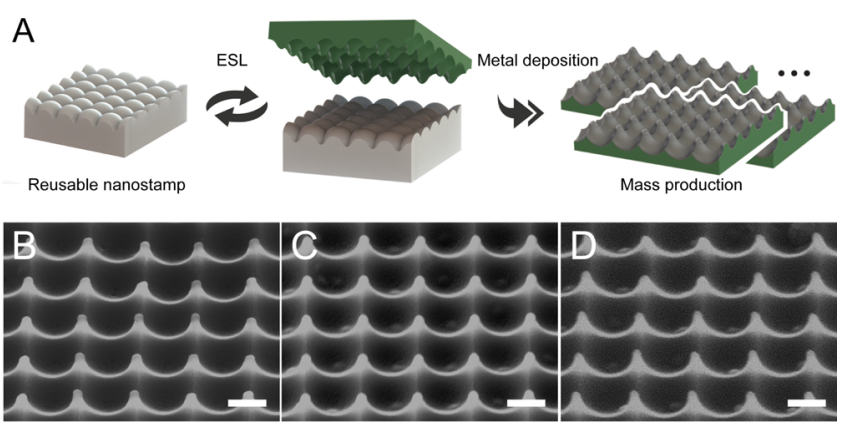

Figure 1. (A) Fabrication procedure of the NPA using ESL and subsequent metal thermal evaporation. (B-D) Side-view SEM images of $\mathrm{Al} \mathrm{NPAs}$ at $45^{\circ}$. These $\mathrm{Al} \mathrm{NPAs}$ were obtained from different batches. The scale bars in (B-D) are $1 \mu \mathrm{m}$.

and subsequent metal thermal evaporation. The as-prepared metal NPA is composed of a transparent polymer substrate and a $50 \mathrm{~nm}$ thick metal deposition. ESL was performed by a polydimethylsiloxane (PDMS) nanostamp and an UV-cured norland optical adhesive (NOA). The PDMS nanostamp is flexible and reusable for more than 50 times, which can reduce the costs for mass production. We are able to measure the transmission spectra of such a NPA because the NOA substrate is transparent, which greatly simplified the detection system. ${ }^{40}$ On the basis of the tapering shape of the nanopyramid, the NPA substrate was easily detached from its complementary nanostamp in the ESL procedure, which enabled the uniformity in a large area and the consistency between different batches. The uniformity of the NPA in a large area $\left(0.25 \mathrm{~cm}^{2}\right)$ is demonstrated in Figure S1, and the consistency of NPAs from five batches in morphologies and optical properties are shown in Figures $1 \mathrm{~B}-\mathrm{D}$ and $\mathrm{S} 2$, respectively. Various types of metal NPAs (Al NPA, Au NPA, and Ag NPA) were prepared by the subsequent thermal evaporation (Figure S3). Figures $1 \mathrm{~B}-\mathrm{D}$ and S4 show the morphologies of NPAs through the $45^{\circ}$ sideview scanning electron microscope (SEM) images and top-view SEM images. The lattice constant of the array $(l)$ is $1.4 \mu \mathrm{m}$, the height of the nanopyramid $(h)$ is $590 \mathrm{~nm}$, and the depth of the nanoconcave $(d)$ is $368 \mathrm{~nm}$. This nanopyramid possessed geometries of nanotips and nanoedges (Figure S4), which enabled the presence of hotspots for high sensitivity of refractometric sensing and SERS. This two-dimensional (2D) NPA features a morphology similar to that of bulk NPA in our previous work. ${ }^{7}$ However, in contrast to the fabrication technique of electrochemical etching used in the previous work, the technique of ESL used here was simple, cost-efficient, and accessible to reproducible NPAs.

Optical Properties of $\mathrm{Al}, \mathrm{Au}$, and Ag NPAs. Figure 2A shows the normal transmission spectra of the as-obtained $\mathrm{Al}$, $\mathrm{Au}$, and $\mathrm{Ag}$ NPAs in air at the wavelength region from 400 to $1100 \mathrm{~nm}$. The transmission spectra of corresponding metal flat films were also measured as references (Figure 2B). Multiple characteristic peaks were observed on the spectra of metal NPAs in contrast to those of flat films. Our previous work of bulk Al NPAs with similar morphologies displayed several LSPR peaks in the whole UV-visible-infrared region as well. The troughs at $700 \mathrm{~nm}$ and at $\sim 990 \mathrm{~nm}$ (equals to $\frac{1}{2} l$ and $\frac{1}{2} \sqrt{2} l$, respectively) of all three metal NPAs mainly resulted from the second-order Rayleigh diffraction anomaly between the adjacent nanopyramids and between the diagonally adjacent nanopyramids, respectively. ${ }^{41}$ It is noteworthy mentioning that 

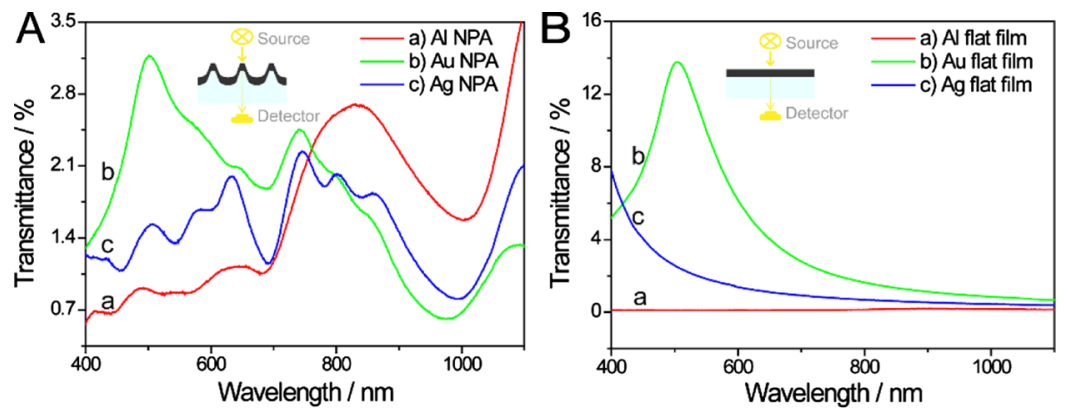

Figure 2. Transmission spectra of $\mathrm{Al}, \mathrm{Au}$, and $\mathrm{Ag}$ NPAs (A) and flat films (B) exposed in air (curve a for $\mathrm{Al}$, curve b for $\mathrm{Au}$, and curve $\mathrm{c}$ for $\mathrm{Ag}$ ). The insets show the perpendicular transmission system employed in the sensing process.
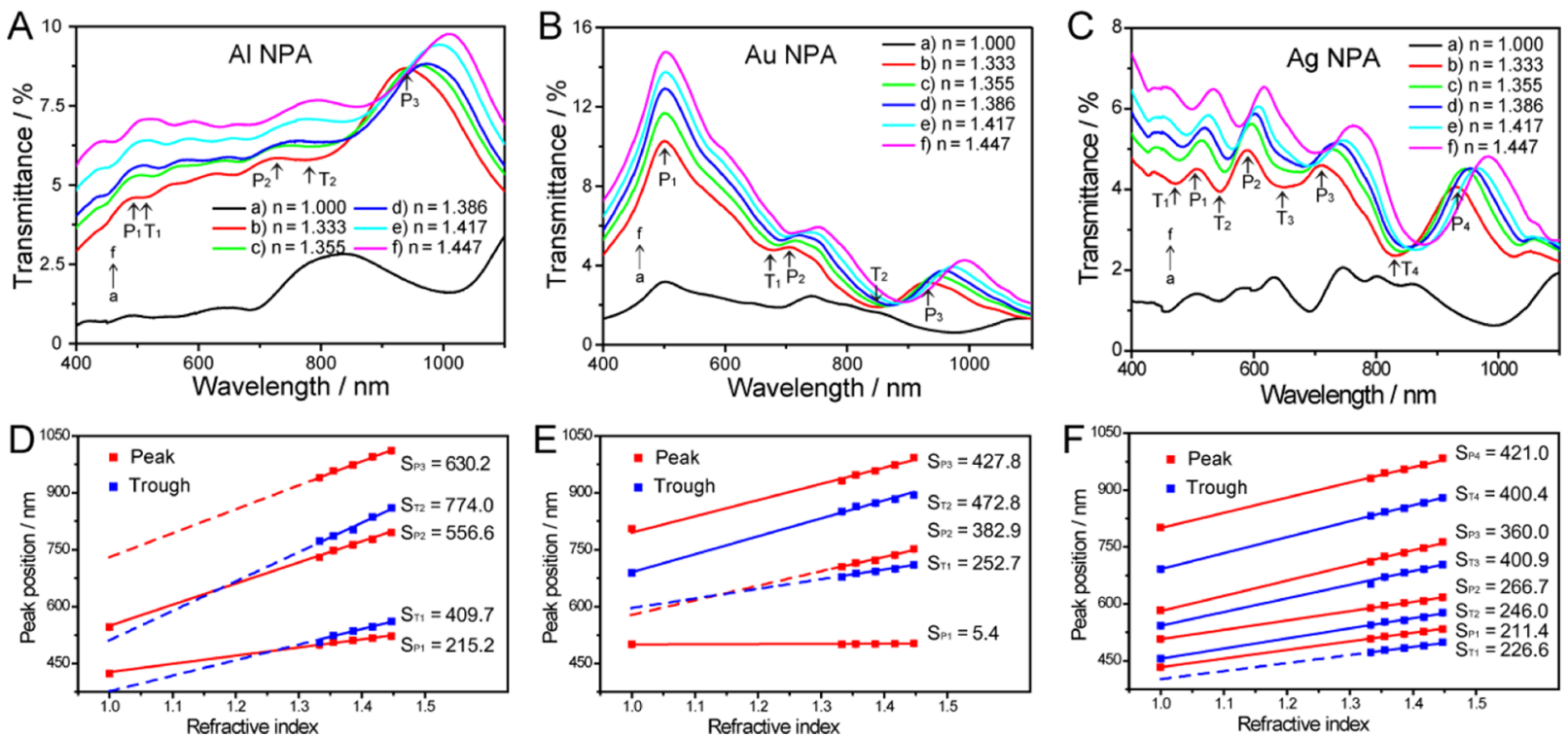

Figure 3. Refractometric sensitivities of $\mathrm{Al}, \mathrm{Au}$, and $\mathrm{Ag}$ NPAs. (A-C) Transmission spectra of $\mathrm{Al}, \mathrm{Au}$, and $\mathrm{Ag}$ NPAs when the superstrates were air and glycerol/water mixtures with different RIs ( $n$ ), respectively. (D-F) Relationships between the position of the peak/trough and corresponding $n$ of the superstrate. The troughs and peaks are arrowed in $(\mathrm{A}-\mathrm{C})$. The slopes of the linear fitting curves indicate the refractometric sensitivities, whose unit is nm/RIU. Error bars for each point are too small to been seen.

the peak of the Al NPA at $827 \mathrm{~nm}$ located in the region of biooptical window $(700-1100 \mathrm{~nm})$, which made it a promising candidate for biosensing involving tissues and cells. For both $\mathrm{Au}$ NPA (curve b, Figure 2A) and Au flat film (curve b, Figure $2 \mathrm{~B}$ ), the large resonance peak at $502 \mathrm{~nm}$ was the characteristic PSPR peak. ${ }^{42}$ All of these peaks here presented relatively narrow bandwidth, in which the smallest full width at halfmaximum was $21.7 \mathrm{~nm}$ from the $\mathrm{Ag} \mathrm{NPA}$, benefiting the refractometric sensing of NPAs based on the LSPR peak shift. ${ }^{43}$

Refractometric Sensitivities of $\mathrm{Al}, \mathrm{Au}$, and Ag NPAs. We have measured the transmission spectra of NPAs when they were covered by superstrates with different RIs. The superstrates were air $(n=1)$ and glycerol/water mixtures with different ratios $(n=1.333,1.355,1.386,1.417$, and 1.447). A normal transmission optical path was used during the measurements to eliminate systematic errors caused by the RI variation of superstrates. ${ }^{39}$ As the RI increased, the resonance peaks and troughs of the NPAs red shifted and the transmittance increased as well, as shown in Figure 3A-C. The relationships between the peak/trough position and the corresponding $\mathrm{RI}(n)$ for $\mathrm{Al}, \mathrm{Au}$, and $\mathrm{Ag}$ NPAs were exhibited in Figure 3D-F, respectively. Linear fitting results of these relationships were demonstrated with high goodness of fit $\left(R^{2}\right.$ $>0.99$ ), and the slopes of the linear fitting curves indicated the refractometric sensitivities. The refractometric sensitivities of these peaks tended to augment with the increase of the peak wavelength, and the refractometric sensitivities of these troughs also exhibited the similar phenomenon (Figure S5). Notably, high refractometric sensitivities of 774.0, 472.8, and $421.0 \mathrm{~nm} /$ RIU were observed on $\mathrm{Al}, \mathrm{Au}$, and $\mathrm{Ag}$ NPAs, respectively. A summary of $\mathrm{Al}$ nanostructures for refractometric biosensing is provided in Table S1. To our best knowledge, this refractometric sensitivity of $774 \mathrm{~nm} /$ RIU was the highest one among those of $\mathrm{Al}$ nanoparticles and $2 \mathrm{D} \mathrm{Al}$ nanostructures. In addition, it is closed to the refractometric sensitivity of the bulk Al NPA (819 nm/RIU). ${ }^{7}$ Besides the similar high sensitivity, this 2D Al NPA exceeded the bulk Al NPA in the massproducing procedure based on the high reproducibility and the cost efficiency of its preparing methods. These advantages made the 2D NPA a competing candidate as LSPR biosensor for practically biomedical applications.

Semiquantitative Detection of Cell Concentration by the Al NPA. Monitoring the proliferation of cancer cells is significant for investigating the growth, infiltration, recurrence, and migration of tumors. We integrated the as-prepared $\mathrm{Al}$ NPA with a high refractometric sensitivity into a LSPR sensing platform for monitoring the proliferation of HeLa cancer cells in situ and in real time. To avoid the influence of the optical 

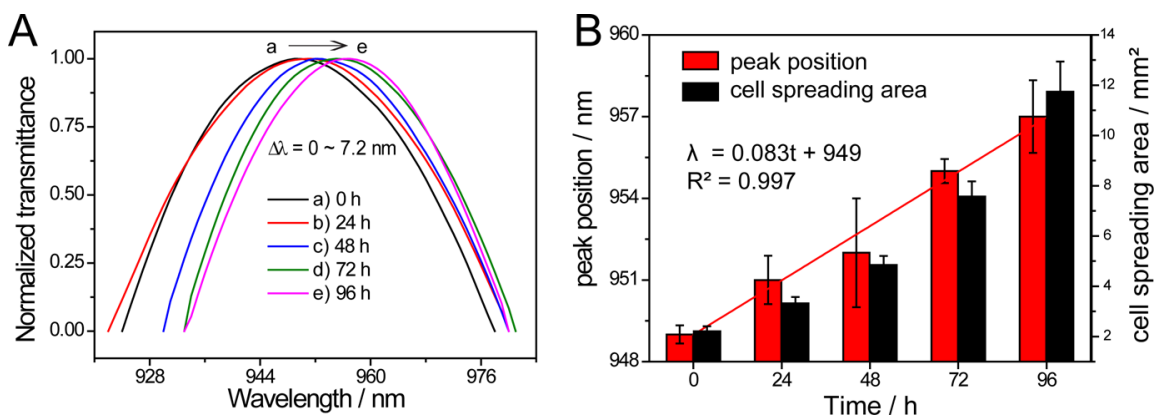

Figure 4. (A) Normalized transmission spectra of $\mathrm{P}_{3}$ for an Al NPA biosensor when monitoring HeLa cell's proliferation for $0,24,48,72$, and $96 \mathrm{~h}$ (curve a to curve e). The maximal peak shift $\left(\Delta \lambda_{\max }\right)$ is about $7.2 \mathrm{~nm}$. (B) Relationship between the LSPR peak position and the proliferation time (red columns) and the relationship between the cell spreading area and the proliferation time (black columns). The red line indicates the linear relationship between the peak position and time. Using the Pearson correlation coefficient, a significant correlation was observed between the cell spreading area and the LSPR peak position $(r=0.979, P=0.004)$.
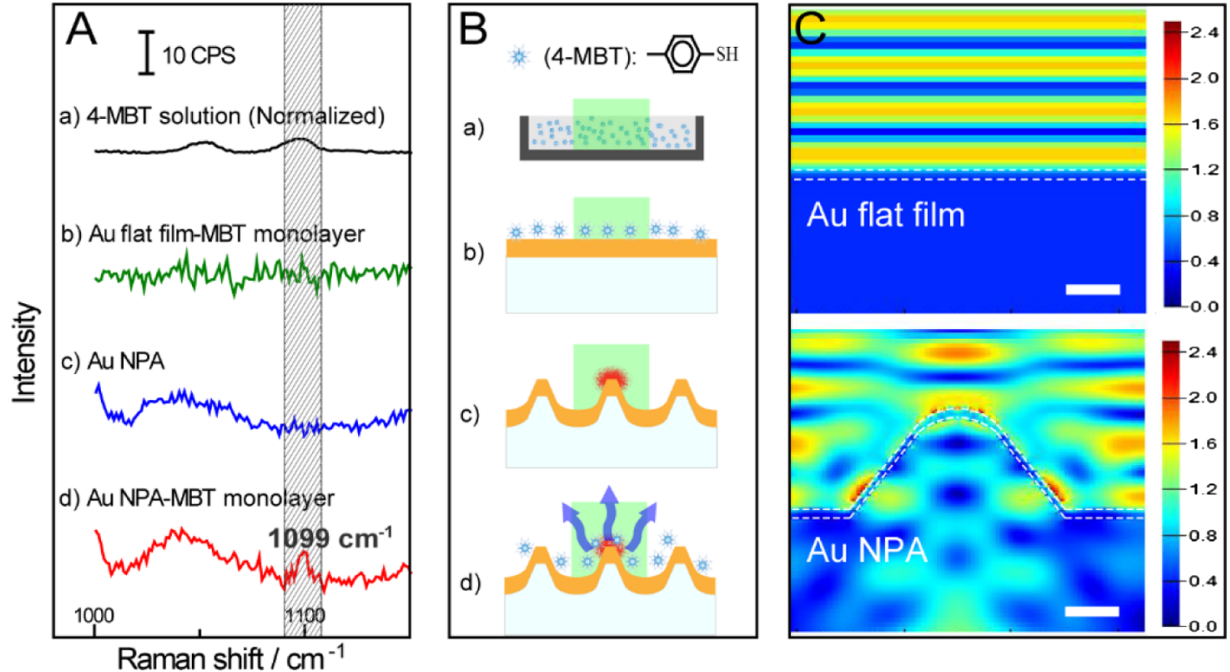

Figure 5. (A) Raman spectra of the 4-MBT solution (normalized, curve a), a Au flat film adsorbed with 4-MBT (curve b), a bare Au NPA (curve c), and a Au NPA adsorbed with 4-MBT (curve d). A peak at $1099 \mathrm{~cm}^{-1}$ was observed at the Raman scattering spectra of the 4-MBT solution and the Au NPA adsorbed with 4-MBT. (B) Schematic diagrams illustrating the structure of 4-MBT and the Raman scattering process of the 4-MBT solution (a), a Au flat film adsorbed with the 4-MBT monolayer (b), a bare Au NPA (c), and a Au NPA adsorbed with the 4-MBT monolayer (d). (C) FDTD simulations of EF distribution for the Au flat film and the Au NPA with light at normal incidence. The dashed lines and curves outline the $50 \mathrm{~nm}$ thick $\mathrm{Au}$ layers of the Au flat film and the Au NPA. EF enhancement was observed at the nanotip and the nanoedge. The scale bars in (C) are $200 \mathrm{~nm}$.

absorption spectra of cellular components, $\mathrm{P}_{3}$ at the infrared region with a high refractometric sensitivity $(630.2 \mathrm{~nm} / \mathrm{RIU})$ was used for the measurements. Healthy HeLa cells with controlled amount ( 30000 cells per $\mathrm{mL}$ ) were seeded on the $\mathrm{Al}$ NPA and constantly proliferated for $96 \mathrm{~h}$ without fully covering the metal surface. The cells have higher RI $\left(n_{\text {cytoplasm }}=1.38\right.$, $\left.n_{\text {membrane }}=1.48\right)$ than that in the culture medium $\left(n_{\text {medium }}=\right.$ $1.33)$. With the proliferation of cells, increasing cells replaced the culture medium and occupied more sensing area of the $\mathrm{Al}$ NPA. As a result, the RI near the surface of the Al NPA increased constantly. In addition, this variation of the local RI was demonstrated by the shift of the LSPR peak. As shown in Figure 4A, transmission spectra (from 920 to $990 \mathrm{~nm}$ ) of this $\mathrm{Al}$ NPA were measured every $24 \mathrm{~h}$. In addition, the resonance peak red shifted for $\sim 7.2 \mathrm{~nm}$ (from 949.4 to $956.6 \mathrm{~nm}$ ) in $96 \mathrm{~h}$. A linear relationship between the peak position $(\lambda)$ and time (t) was observed (Figure 4B, red line). Micrographs of the cells were recorded at the same time points (Figure S6), to certify the enlarging amount of cells and the cells partly covering the NPA. From the micrographs, we recorded the cell spreading area at different times (Figure $4 \mathrm{~B}$, black columns). Using the Pearson correlation coefficient, a significant correlation was observed between the cell spreading area and the LSPR peak shift $(r=0.979, P=0.004)$.

Cell proliferation can be detected by other experimental methods (such as microscope, piezoelectric sensing, electric cell-substrate impedance sensing, and surface plasmon resonance sensing). However, our method based on the LSPR biosensor has two advantages compared to the above methods. The equipment that we need is a spectrometer, which is lowcost. In addition, information such as cell-substrate interaction is able to be obtained by LSPR sensors while it is not provied by other sensors. Since the LSPR sensor is sensitive to the RI in the vicinity of the metal surface, only the alive cells contact closely to the substrate and induce the LSPR signals, whereas the dead cells suspend in the culture medium without interfering the signals. Therefore, our LSPR sensors mainly obtained the signals caused by the alive cells on the sensor surface. 
Qualitative Identification of Molecules on the $\mathrm{Au}$ NPA. The enhanced localized EF induced by LSPR has not only been utilized in refractometric biosensing but also in surface-enhanced assays such as surface-enhanced fluorescence $^{44}$ and SERS. ${ }^{45-47}$ SERS can provide a structural fingerprint of an analyte, which can be applied to identifying the molecules functioning in the process of the cell-substrate interaction. ${ }^{48,49}$ Here, we demonstrate the capacity of the NPA to work as a SERS substrate. We immersed the Au NPA substrate in a solution of the model analyte, 4-MBT. This molecule was adsorbed on the NPA based on the strong $\mathrm{Au}-\mathrm{S}$ linkage between the molecule and the Au surface. Figure 5A shows the Raman spectra of 4-MBT solution (normalized, curve a), 4-MBT monolayer-absorbed Au flat film (curve b), bare $\mathrm{Au}$ NPA (curve c), and 4-MBT monolayer-absorbed $\mathrm{Au}$ NPA (curve d). The characteristic peak at $\sim 1100 \mathrm{~cm}^{-1}$ was attributed to a combination of the phenyl ring breathing mode, $\mathrm{CH}$ in-plane bending, and CS stretching. ${ }^{50}$ There was no peak appeared at around $1100 \mathrm{~cm}^{-1}$ on the Raman spectrum of the 4-MBT-absorbed Au flat film, whereas an enhance peak at 1099 $\mathrm{cm}^{-1}$ was observed on the Raman spectrum of the 4-MBTabsorbed Au NPA. A schematic diagram in Figure 5B indicates how 4-MBT absorbed on the Au flat film (scheme b) and the $\mathrm{Au}$ NPA (scheme d), and then responded to the Raman exciting laser. Compared to the flat film, there were hotspots (localized enhanced EF) induced by LSPRs occurring on the nanotips and nanoedges of NPAs; therefore, these hotspots enhanced the Raman scattering signals.

To investigate the mechanism of SERS capacity and high refractometric sensitivity of NPAs, we have simulated the EF distribution of the Au flat film and the Au NPA by using the FDTD solutions (Figure 5C). Band-shaped EF distribution was observed above the Au flat film, resulting from the interference between the incident and reflected light. In contrast, localized hotspots at both tip and edges of the nanopyramid were attained on the Au NPA. The localization and intensification of EF on the NPA can improve the performance of the LSPR biosensor for refractometric and surface-enhanced Raman biosensing.

In conclusion, we have prepared a type of plasmonic NPA with excellent reproducibility (large-area uniformity and batchto-batch consistency) by using ESL and metal thermal evaporation, which obtained high refractometric sensitivity based on hotspots at nanotips and nanoedges. Specific detection of molecules such as cancer biomarkers or thrombin could be achieved on such a LSPR biosensor after chemical modification. ${ }^{7,51}$ In addition, the Au NPA-based SERS illustrated that this NPA LSPR nanosubstrate is capable to be applied in molecular identification, such as the detection of glucose. ${ }^{52}$ In comparison to the existing LSPR biosensors, our proposed work exhibit the following advantages: (1) the NPA with the geometry of the tapering nanostructure is very suitable for ESL. Compared to the nanopillar and nanomushroom, a nanopyramid is much easier to be detached from its complementary nanostamp without damage. This characteristic makes the NPA biosensor prepared by ESL approaches highly reproducible, which indicates the remarkable reliability of LSPR biosensors for practical use; (2) the fabricating technique combining ESL and metal thermal evaporation has provided various types of metal NPAs (e.g., $\mathrm{Al}, \mathrm{Au}$, and $\mathrm{Ag}$ NPAs), meeting the demands of different situations. This fabrication technique could also utilize other non-noble metal materials such as $\mathrm{Cu}$ to fabricate NPAs. Moreover, these non-noble metal
NPAs including Al NPA with properties of low cost, ease to process, and abundant mineral reserve have great potential in constructing high-performance LSPR biosensors; (3) the NPAs with deliberately designed sharp tips and edges confine light into nanoscale regions and create dense hotspots for highly sensitive refractometric biosensing and surface-enhanced spectroscopies, certifying the potential of the NPAs for multiplex applications; (4) the NPA will enable the combination of quantitative LSPR sensing and qualitative SERS analysis, where the transmittance changes of UV-visible spectra (light through the NPA) that involve the information of the concentrations and the Raman scattering signals (light backward from the NPA surface) reveals the molecular structure. Considering the simplicity and cost efficiency of the fabrication method, as well as the properties of excellent reproducibility and high sensitivity, this NPA LSPR substrate will accelerate the process of applying the LSPR sensor into practical use such as clinical diagnosis, home healthcare, environmental analysis, and public healthcare.

\section{METHODS}

Materials. PDMS (SYLGARD 184) was purchased from Dow Corning (US). NOA61 was obtained from Norland Products (US). Gold, silver, and aluminum particles for metal thermal evaporation were from ZhongNuo Advanced Material Technology Company (Beijing, China). Ethanol and glycerol were supplied by Damao chemical reagent (Tianjin, China). For cells' experiments, the Dulbecco's modified Eagle's medium was purchased from HyClone (US). Bovine serum was from Gibco (Singapore). For SERS experiments, 4-MBT was obtained from Aladdin (Shanghai, China). All chemicals and solvents used were of analytical grade and were used as received. Deionized water was used for all experiments.

Fabrication of Metal NPAs. A bulk Al NPA template was previously fabricated through denting and subsequent electrochemical etching on a flat aluminum sheet. ${ }^{7} \mathrm{~A}$ well-mixed liquid PDMS prepolymer was poured onto the template, degassed, and solidified in an oven $\left(80^{\circ} \mathrm{C}, 30 \mathrm{~min}\right)$. The solidified PDMS nanostamp was then peeled off from the template. During the next ESL process, the solution was dripped onto the PDMS nanostamp. To increase the sharpness of the nanopyramid, a type of NOA61 solution with lower viscosity was employed and a $12 \mathrm{~h}$ standing in the vacuum was operated. The NOA61 substrate was then solidified under UV light (Kinsten KVB30D, Taiwan) (15 W, $5 \mathrm{~min}$ ) and peeled off. After that, $50 \mathrm{~nm}$ thick $\mathrm{Al}, \mathrm{Au}$, or $\mathrm{Ag}$ layers were deposited onto the NOA61 substrate through thermal evaporation.

It is worth mentioned that a stable native oxide layer of aluminum on the Al NPA was formed in hours, with a thickness stabilizing at $2.5-3 \mathrm{~nm} .{ }^{53}$ In addition, the workability of the passivated aluminum nanostructure as an optical biosensor has also been proved. ${ }^{54}$

Characterization of NPAs and Nanofilms. SEM images of metal NPAs were captured by a SEM (Zeiss Auriga). The transmission spectra of $\mathrm{Al}, \mathrm{Au}$, and $\mathrm{Ag}$ NPAs under different superstrates of air $(n=1)$ and glycerol/water mixtures were measured using a UV-visible spectrophotometer (Inesa L3S). The mixing ratios of the above mixtures are $0,25.96,41.46$, 62.50, and 86.57 wt \%, whereas the according RIs are 1.333, $1.355,1.386,1.417$, and 1.447 , respectively. To determine the standard deviation (SD) of the peak position, three measurements were performed at each RI. The appearance of the NPA chip was captured by cell phone (iPhone 5S). 
In the cell experiment, the Al NPA was previously immobilized on the inner wall of the cell culture flask. The whole flask was then sterilized and washed by phosphatebuffered saline. Transmission spectra of the NPA biosensor were measured every $24 \mathrm{~h}$ after healthy HeLa cancer cells were seeded on the surface of the nanostructure. To determine the SD of the peak position, three measurements were performed at each time. In the meantime, micrographs of cells on the $\mathrm{Al}$ NPA were taken from an inverted fluorescence microscope (Nikon Eclipse Ti-U). The area of each micrograph is 1/300 of the area of Al NPA. We counted the pixel points in the micrograph and multiplied 300 to estimate the whole cell spreading area on Al NPA.

In the SERS experiment, the Au NPA and the Au film were both immersed in the 4-MBT/ethanol solution (0.05 M, $3 \mathrm{~mL}$ ) in a Petri dish $(\Phi=3.5 \mathrm{~cm})$ for $15 \mathrm{~min}$, respectively. Then, they were washed by ethanol and dried in air. The Raman spectra of $0.05 \mathrm{M}$ 4-MBT solution, 4-MBT-adsorbed Au film, bare $\mathrm{Au}$ NPA, and 4-MBT-adsorbed Au NPA were measured on a Raman spectrometer (Renishaw inVia Reflex). The Raman spectrum of the 4-MBT solution (0.05 M) was normalized for further analysis. The wavelength of the excitation laser was 532 $\mathrm{nm}$. The laser power and signal acquisition time were $1 \mathrm{~mW}$ and $10 \mathrm{~s}$, respectively.

FDTD Simulation. The EF distribution of the $\mathrm{Au}$ film and the Au NPA under a $532 \mathrm{~nm}$ light source was simulated using FDTD solutions (Lumerical Solutions). The NPA was simplified as a combination of two vertical gradient grating arrays and one nanocone array. All of the dimension parameters were set according to the SEM images. Mesh size for the metal region was $3 \mathrm{~nm}(x$-, $y$-, and $z$-directions). The RI of NOA61 was 1.56 .

\section{ASSOCIATED CONTENT}

\section{S Supporting Information}

The Supporting Information is available free of charge on the ACS Publications website at DOI: 10.1021/acsomega.7b02016.

SEM image showing the large-area uniformity of an $\mathrm{Al}$ NPA chip; transmission spectra showing the batch-tobatch consistency of optical properties of NPAs; SEM images of Al NPA, Au NPA, and Ag NPA; top-view SEM image of Al NPA and magnification image of a single nanopyramid; the increasing tendency of refractometric sensitivity with a peak or trough position; microscopic images of cell proliferation; and summary of aluminum nanostructures for refractometric biosensing (PDF)

\section{AUTHOR INFORMATION}

\section{Corresponding Authors}

*E-mail: lixm67@mail.sysu.edu.cn. Phone: +86 2039332150. (X.L.).

*E-mail: zhoujh33@mail.sysu.edu.cn. Phone: +8620 39387890. Fax: +86 20 39387890. (J.Z.).

\section{ORCID 1}

Huanjun Chen: 0000-0003-4699-009X Jianhua Zhou: 0000-0001-9895-8679

\section{Notes}

The authors declare no competing financial interest.

\section{ACKNOWLEDGMENTS}

This work was supported in part by the Ministry of Science and Technology of China (2017YFE0102400), National Natural Science Foundation of China (no. 21775168), Department of Science and Technology of Guangdong Province (2017A020211004), and the special support plan for training high-level talents in Guangdong Province (no. 2014TQ01R695). The work was also supported in part by the Australia-China Joint Institute for Health Technology and Innovation.

\section{REFERENCES}

(1) Liu, L.; Gao, P.; Liu, K.; Kong, W.; Zhao, Z.; Pu, M.; Wang, C.; Luo, X. Nanofocusing of circularly polarized Bessel-type plasmon polaritons with hyperbolic metamaterials. Mater. Horiz. 2017, 4, 290296.

(2) Kabashin, A. V.; Evans, P.; Pastkovsky, S.; Hendren, W.; Wurtz, G. A.; Atkinson, R.; Pollard, R.; Podolskiy, V. A.; Zayats, A. V. Plasmonic nanorod metamaterials for biosensing. Nat. Mater. 2009, 8, $867-871$.

(3) Wu, K.; Chen, J.; McBride, J. R; Lian, T. Efficient hot-electron transfer by a plasmon-induced interfacial charge-transfer transition. Science 2015, 349, 632-635.

(4) Li, J.; Cushing, S. K.; Meng, F.; Senty, T. R.; Bristow, A. D.; Wu, $\mathrm{N}$. Plasmon-induced resonance energy transfer for solar energy conversion. Nat. Photonics 2015, 9, 601-607.

(5) Wang, H.; You, T.; Shi, W.; Li, J.; Guo, L. Au/TiO2/Au as a plasmonic coupling photocatalyst. J. Phys. Chem. C 2012, 116, 64906494.

(6) Xue, J.; Zhou, Z.-K.; Wei, Z.; Su, R.; Lai, J.; Li, J.; Li, C.; Zhang, T.; Wang, X.-H. Scalable, full-colour and controllable chromotropic plasmonic printing. Nat. Commun. 2015, 6, 8906.

(7) Li, W.; Qiu, Y.; Zhang, L.; Jiang, L.; Zhou, Z.; Chen, H.; Zhou, J. Aluminum nanopyramid array with tunable ultraviolet-visible-infrared wavelength plasmon resonances for rapid detection of carbohydrate antigen 199. Biosens. Bioelectron. 2016, 79, 500-507.

(8) Mayer, K. M.; Hafner, J. H. Localized surface plasmon resonance sensors. Chem. Rev. 2011, 111, 3828-3857.

(9) Tang, L.; Li, J. Plasmon-based colorimetric nanosensors for ultrasensitive molecular diagnostics. ACS Sens. 2017, 2, 857-875.

(10) Bharadwaj, R; Mukherji, S.; Mukherji, S. Probing the localized surface plasmon field of a gold nanoparticle-based fibre optic biosensor. Plasmonics 2015, 11, 753-761.

(11) Park, J.-H.; Byun, J.-Y.; Shim, W.-B.; Kim, S. U.; Kim, M.-G. High-sensitivity detection of ATP using a localized surface plasmon resonance (LSPR) sensor and split aptamers. Biosens. Bioelectron. 2015, $73,26-31$.

(12) Joshi, G. K.; Deitz-McElyea, S.; Liyanage, T.; Lawrence, K.; Mali, S.; Sardar, R.; Korc, M. Label-free nanoplasmonic-based short noncoding RNA sensing at attomolar concentrations allows for quantitative and highly specific assay of microRNA-10b in biological fluids and circulating exosomes. ACS Nano 2015, 9, 11075-11089.

(13) Liu, Z.; Yu, M.; Huang, S.; Liu, X.; Wang, Y.; Liu, M.; Pan, P.; Liu, G. Enhancing refractive index sensing capability with hybrid plasmonic-photonic absorbers. J. Mater. Chem. C 2015, 3, 4222-4226.

(14) Haes, A. J.; Van Duyne, R. P. A unified view of propagating and localized surface plasmon resonance biosensors. Anal. Bioanal. Chem. 2004, 379, 920-930.

(15) Willets, A. K.; Van Duyne, R. P. Localized surface plasmon resonance spectroscopy and sensing. Annu. Rev. Phys. Chem. 2007, 58, 267-297.

(16) Estevez, M.-C.; Otte, M. A.; Sepulveda, B.; Lechuga, L. M. Trends and challenges of refractometric nanoplasmonic biosensors: A review. Anal. Chim. Acta 2014, 806, 55-73.

(17) Rampazzi, S.; Danese, G.; Leporati, F.; Marabelli, F. A localized surface plasmon resonance-based portable instrument for quick on-site 
biomolecular detection. IEEE Trans. Instrum. Meas. 2016, 65, 317327.

(18) Joshi, G. K.; Deitz-McElyea, S.; Johnson, M.; Mali, S.; Korc, M.; $\mathrm{Sa}, \mathrm{R}$. Highly specific plasmonic biosensors for ultrasensitive microRNA Detection in plasma from pancreatic cancer patients. Nano Lett. 2014, 14, 6955-6963.

(19) Wang, Z.; Hu, J.; Jin, Y.; Yao, X.; Li, J. In situ amplified chemiluminescent detection of DNA and immunoassay of IgG using special-shaped gold nanoparticles as label. Clin. Chem. 2006, 52, 1958-1961.

(20) Luo, C.; Wang, Y.; Li, X.; Jiang, X.; Gao, P.; Sun, K.; Zhou, J.; Zhang, Z.; Jiang, Q. An optical sensor with polyaniline-gold hybrid nanostructures for monitoring $\mathrm{pH}$ in saliva. Nanomaterials 2017, 7, 67.

(21) Wei, H.; Abtahi, S. M. H.; Vikesland, P. J. Plasmonic colorimetric and SERS sensors for environmental analysis. Environ. Sci.: Nano 2015, 2, 120-135.

(22) Chu, W.; Zhang, Y.; Li, D.; Barrow, C. J.; Wang, H.; Yang, W. A biomimetic sensor for the detection of lead in water. Biosens. Bioelectron. 2015, 67, 621-624.

(23) Verellen, N.; Van Dorpe, P.; Huang, C.; Lodewijks, K.; Vandenbosch, G. A. E.; Lagae, L.; Moshchalkov, V. V. Plasmon line shaping using nanocrosses for high sensitivity localized surface plasmon resonance sensing. Nano Lett. 2011, 11, 391-397.

(24) Chen, H.; Kou, X.; Yang, Z.; Ni, W.; Wang, J. Shape- and sizedependent refractive index sensitivity of gold nanoparticles. Langmuir 2008, 24, 5233-5237.

(25) Katyal, J.; Soni, R. K. Size- and shape-dependent plasmonic properties of aluminum nanoparticles for nanosensing applications. $J$. Mod. Opt. 2013, 60, 1717-1728.

(26) Lee, K.-S.; El-Sayed, M. A. Gold and silver nanoparticles in sensing and imaging: Sensitivity of plasmon response to size, shape, and metal composition. J. Phys. Chem. B 2006, 110, 19220-19225.

(27) Flauraud, V.; Mastrangeli, M.; Bernasconi, G. D.; Butet, J.; Alexander, D. T. L.; Shahrabi, E.; Martin, O. J. F.; Brugger, J. Nanoscale topographical control of capillary assembly of nanoparticles. Nat. Nanotechnol. 2016, 12, 73-80.

(28) Shen, Y.; Zhou, J.; Liu, T.; Tao, Y.; Jiang, R.; Liu, M.; Xiao, G.; Zhu, J.; Zhou, Z.-K.; Wang, X.; Jin, C.; Wang, J. Plasmonic gold mushroom arrays with refractive index sensing figures of merit approaching the theoretical limit. Nat. Commun. 2013, 4, 2381.

(29) Oh, Y.-J.; Kang, M.; Park, M.; Jeong, K.-H. Engineering hot spots on plasmonic nanopillar arrays for SERS: A review. BioChip J. 2016, 10, 297-309.

(30) Tsai, C.-Y.; Lin, J.-W.; Wu, C.-Y.; Lin, P.-T.; Lu, T.-W.; Lee, P.T. Plasmonic coupling in gold nanoring dimers: Observation of coupled bonding mode. Nano Lett. 2012, 12, 1648-1654.

(31) Lin, D.; Wu, Z.; Li, S.; Zhao, W.; Ma, C.; Wang, J.; Jiang, Z.; Zhong, Z.; Zheng, Y.; Yang, X. Large-area Au-nanoparticle-functionalized Si Nanorod Arrays for Spatially Uniform Surface-enhanced Raman Spectroscopy. ACS Nano 2017, 11, 1478-1487.

(32) Chen, H.; Shao, L.; Li, Q.; Wang, J. Gold nanorods and their plasmonic properties. Chem. Soc. Rev. 2013, 42, 2679-2724.

(33) Okazaki, S. High resolution optical lithography or high throughput electron beam lithography: The technical struggle from the micro to the nano-fabrication evolution. Microelectron. Eng. 2015, $133,23-35$.

(34) Fan, M.; Andrade, G. F. S.; Brolo, A. G. A review on the fabrication of substrates for surface enhanced Raman spectroscopy and their applications in analytical chemistry. Anal. Chim. Acta 2011, 693, $7-25$.

(35) Wen, X.; Datta, A.; Traverso, L. M.; Pan, L.; Xu, X.; Moon, E. E. High throughput optical lithography by scanning a massive array of bowtie aperture antennas at near-field. Sci. Rep. 2015, 5, 16192.

(36) Chen, K.; Rajeeva, B. B.; Wu, Z.; Rukavina, M.; Dao, T. D.; Ishii, S.; Aono, M.; Nagao, T.; Zheng, Y. Moiré nanosphere lithography. ACS Nano 2015, 9, 6031-6040.

(37) Candeloro, P.; Iuele, E.; Perozziello, G.; Coluccio, M. L.; Gentile, F.; Malara, N.; Mollace, V.; Fabrizio, E. D. Plasmonic nanoholes as SERS devices for biosensing applications: An easy route for nanostructures fabrication on glass substrates. Microelectron. Eng. 2017, 175, 30-33.

(38) Im, H.; Lee, S. H.; Wittenberg, N. J.; Johnson, T. W.; Lindquist, N. C.; Nagpal, P.; Norris, D. J.; Oh, S.-H. Template-stripped smooth $\mathrm{Ag}$ nanohole arrays with silica shells for surface plasmon resonance biosensing. ACS Nano 2011, 5, 6244-6253.

(39) Li, W.; Xue, J.; Jiang, X.; Zhou, Z.; Ren, K.; Zhou, J. Low-cost replication of plasmonic gold nanomushroom arrays for transmissionmode and multichannel biosensing. RSC Adv. 2015, 5, 61270-61276.

(40) Cappi, G.; Spiga, F. M.; Moncada, Y.; Ferretti, A.; Beyeler, M.; Bianchessi, M.; Decosterd, L.; Buclin, T.; Guiducci, C. Label-free detection of tobramycin in serum by transmission-localized surface plasmon resonance. Anal. Chem. 2015, 87, 5278-5285.

(41) Liu, F.; Zhang, X. Fano coupling between Rayleigh anomaly and localized surface plasmon resonance for sensor applications. Biosens. Bioelectron. 2015, 68, 719-725.

(42) Peer, A.; Biswas, R. Extraordinary optical transmission in nanopatterned ultrathin metal films without holes. Nanoscale 2016, 8, 4657-4666.

(43) Liu, Z.; Liu, G.; Huang, S.; Liu, X.; Pan, P.; Wang, Y.; Gu, G. Multispectral spatial and frequency selective sensing with ultracompact cross-shaped antenna plasmonic crystals. Sens. Actuators, B 2015, 215, 480-488.

(44) Fort, E.; Grésillon, S. Surface enhanced fluorescence. J. Phys. D: Appl. Phys. 2008, 41, 013001.

(45) Hu, J.; Wang, Z.; Li, J. Gold nanoparticles with special shapes: Controlled synthesis, surface-enhanced Raman scattering, and the application in biodetection. Sensors 2007, 7, 3299-3311.

(46) Shen, Y.; Cheng, X.; Li, G.; Zhu, Q.; Chi, Z.; Wang, J.; Jin, C. Highly sensitive and uniform surface-enhanced Raman spectroscopy from grating-integrated plasmonic nanograss. Nanoscale Horiz. 2016, 1, 290-297.

(47) Liu, G.-q.; Yu, M.-d.; Liu, Z.-q.; Liu, X.-s.; Huang, S.; Pan, P.-p.; Wang, Y.; Liu, M.-1.; Gu, G. One-process fabrication of metal hierarchical nanostructures with rich nanogaps for highly-sensitive surface-enhanced Raman scattering. Nanotechnology 2015, 26, 185702.

(48) Zrimsek, A. B.; Chiang, N.; Mattei, M.; Zaleski, S.; McAnally, M. O.; Chapman, C. T.; Henry, A.-I.; Schatz, G. C.; Van Duyne, R. P. Single-molecule chemistry with surface- and tip-enhanced Raman spectroscopy. Chem. Rev. 2017, 117, 7583-7613.

(49) Jiang, S.; Zhang, Y.; Zhang, R.; Hu, C.; Liao, M.; Luo, Y.; Yang, J.; Dong, Z.; Hou, J. G. Distinguishing adjacent molecules on a surface using plasmon-enhanced Raman scattering. Nat. Nanotechnol. 2015, $10,865-869$.

(50) Liu, P.; Chen, H.; Wang, H.; Yan, J.; Lin, Z.; Yang, G. Fabrication of $\mathrm{Si} / \mathrm{Au}$ core/shell nanoplasmonic structures with ultrasensitive surface-enhanced Raman scattering for monolayer molecule detection. J. Phys. Chem. C 2015, 119, 1234-1246.

(51) Yan, J.; Wang, L.; Tang, L.; Lin, L.; Liu, Y.; Li, J. Enzyme-guided plasmonic biosensor based on dual-functional nano-hybrid for sensitive detection of thrombin. Biosens. Bioelectron. 2015, 70, 404410.

(52) Chen, Q.; Fu, Y.; Zhang, W.; Ye, S.; Zhang, H.; Xie, F.; Gong, L.; Wei, Z.; Jin, H.; Chen, J. Highly sensitive detection of glucose: A quantitative approach employing nanorods assembled plasmonic substrate. Talanta 2017, 165, 516-521.

(53) Gérard, D.; Gray, S. K. Aluminium plasmonics. J. Phys. D: Appl. Phys. 2015, 48, 184001.

(54) Canalejas-Tejero, V.; Herranz, S.; Bellingham, A.; MorenoBondi, M. C.; Barrios, C. A. Passivated aluminum nanohole arrays for label-free biosensing applications. ACS Appl. Mater. Interfaces 2014, 6, $1005-1010$. 